\title{
Enhancement of the photostability of flavylium dye adsorbed on
}

\author{
mesoporous silicate
}

\begin{abstract}
Yoshiumi Kohno ${ }^{a,}{ }^{*}$, Shunsuke Tsubota ${ }^{a}$, Yoshinori Shibata ${ }^{a}$, Kazuya Nozawa $^{a}$, Keiko Yoda ${ }^{b}$, Masashi Shibata $^{b}$, Ryoka Matsushima ${ }^{a}$

${ }^{a}$ Department of Materials Science and Engineering, Faculty of Engineering, Shizuoka University, 3-5-1
\end{abstract}

Johoku, Hamamatsu, Shizuoka, 432-8561, Japan

${ }^{b}$ Beauty Research Center, Kao Corporation, 2-1-3 Bunka, Sumida-ku, Tokyo 131-8501, Japan

\section{Abstract}

Flavylium dye, a model compound of natural anthocyanin, has a defect that its color stability is very poor. We have tried to complex the flavylium dye with several mesoporous silicas (FSM-16, MCM-41 and HMS) and succeeded to enhance the photostability of the dye against visible light irradiation. We have found that the light fastness is enhanced when the dye is incorporated into the mesoporous silicas exhibiting solid acidity that derives from an aluminum impurity. The solid acidity plays a role to 
stabilize the cationic flavylium dye, while the mesopore structure hinders the attack of oxygen to the incorporated dye molecules. Because of its enhanced color stability, the flavylium dye incorporated in the acidic mesoporous silicas can be a candidate for an environmentally friendly coloring material.

keywords: Flavylium, Anthocyanin, Stabilization, Light fastness, Mesoporous silica 


\section{Introduction}

From ancient times to the present day, organic dyes have been widely used as coloring materials. However, since stability of the organic dyes is often not sufficient, their color is lost by continuous exposure to environment. It has been known that the color stability of the organic dye can be enhanced by hybridization with some inorganic hosts. Even in 8th century, Mayan civilization developed an organic-inorganic hybrid material called Maya Blue, which was a complex of a natural indigo dye and a clay mineral palygorskite [1, 2]. The Maya Blue has kept its color for more than a thousand year even though it has been exposed to a severe climate in a tropical forest. In recent years, many organic dyes are found to be stabilized by hybridization with inorganic hosts such as clays [3-9] or zeolites [9-12]. In addition, there have been some reports concerning the immobilization of organic dyes by incorporation into mesoporous silicates. Fukushima et al. reported that a natural chlorophyll II $a$ was stabilized against light irradiation by incorporation into the mesopore of FSM-16 [13]. One of the present authors reported that the light fastness of an anionic azoic dye was enhanced by 
incorporation into the mesopore of Ca-exchanged MCM-41 [14-16]. Besides, many researchers have reported that complexation with various mesoporous materials can enhance the stability of the incorporated organic dye [17-22].

Flavylium dye (FV) is a cationic dye with a 2-phenylbenzopyrylium skeleton, the same structure as that of naturally occurring anthocyanin dye. Therefore the impact of FV on the environment is expected to be smaller than that of other synthetic dyes [23, 24]. In addition, FV derivatives exhibit three primary colors of yellow, magenta and cyan by selecting proper substituents [25-28]. Accordingly, FV can be a candidate of an environmentally friendly coloring material. However, the color stability of FV against light irradiation, heat or alkaline environment is poor [29-31], as is the natural anthocyanin [32]. If one intends to use FV as a coloring material, the poor stability of $\mathrm{FV}$ is undesirable.

We have already found that complexation of FV with a clay mineral [33] enhances the thermal or chemical stability of the FV. Since in the clay the hydration attack from the exterior is diminished, the room for FV to undergo isomerization is removed, and there exists strong influence of the electrostatic field in the host material on the FV, the FV 
dye is suggested to be stabilized by incorporation in the inorganic host material.

In this study, we tried to enhance the stability of the FV molecule against light irradiation, by incorporating the dye in several mesoporous silicas. Investigations were made about the effect of the mesopore structure, or the amount of included aluminum, on the enhancement of the stability.

\section{Experimental}

We used three mesoporous silicates, FSM-16, HMS and MCM-41, as inorganic host materials. They were synthesized in similar manners described in literatures [34-36].

According to the literature [34], FSM-16 was synthesized from water glass via kanemite. Firstly, sodium hydroxide was added to water glass (Wako Chemical Co., $\mathrm{SiO}_{2} / \mathrm{Na}_{2} \mathrm{O}=2.18, \mathrm{SiO}_{2}=37.0 \mathrm{wt} \%$ ) to let the $\mathrm{Si} / \mathrm{Na}$ ratio unity, and the solution was heated up to $393 \mathrm{~K}$ under reduced pressure. The dried sample was calcined at $973 \mathrm{~K}$ for $6 \mathrm{~h}$ in the air and $\delta-\mathrm{Na}_{2} \mathrm{Si}_{2} \mathrm{O}_{5}$ was obtained. The produced $\delta-\mathrm{Na}_{2} \mathrm{Si}_{2} \mathrm{O}_{5}$ was mixed with water, stirred for $3 \mathrm{~h}$ at room temperature, and filtered to obtain the layered silicate, 
kanemite. The kanemite (4 g) was mixed with the aqueous solution of hexadecyltrimethylammonium bromide (Tokyo Kasei Co., denoted as HDTMABr, 1.82 $\mathrm{g} / 0.8 \mathrm{dm}^{3}$ ) as the molar ratio of HDTMABr/Si became 0.2 , and stirred for $3 \mathrm{~h}$ at $343 \mathrm{~K}$, followed by stirring at room temperature for $12 \mathrm{~h}$. After filtration, the obtained solid was dispersed in $0.6 \mathrm{dm}^{3}$ of water and kept at $343 \mathrm{~K}$ for $6 \mathrm{~h}$ followed by stirring at room temperature for $18 \mathrm{~h}$. Meanwhile, the $\mathrm{pH}$ of the mixture was maintained at 8.5 by adding dilute $\mathrm{HCl}$. The mixture was filtered, and the resulting solid was washed 5 times with $0.6 \mathrm{dm}^{3}$ of water. Finally, the sample was calcined at $823 \mathrm{~K}$ for $6 \mathrm{~h}$ under dry air stream, and FSM-16 was obtained.

HMS was prepared according to the literature [35]. Briefly, $0.908 \mathrm{~g}$ of dodecylamine (Wako) was dissolved in $5 \mathrm{~cm}^{3}$ of ethanol, mixed with $45 \mathrm{~cm}^{3}$ of water, and heated to $333 \mathrm{~K}$. Tetraethoxysilane (Nacalai Tesque Co., denoted as TEOS) weighed $4.6 \mathrm{~g}$ was dropped to the solution, and the mixture was kept at $333 \mathrm{~K}$ for $22 \mathrm{~h}$. The sample was filtered, washed with water, dried in an oven and calcined at $903 \mathrm{~K}$ for $6 \mathrm{~h}$ under dry air stream. The molar composition was 1.0 dodecylamine : 4.5 TEOS : $511 \mathrm{H}_{2} \mathrm{O}: 17$ ethanol. 
MCM-41 was synthesized as follows [36]. An aqueous solution of the surfactant (7.3

mmol) was mixed with $15.34 \mathrm{~g}$ of colloidal silica (Nissan Chemical, Snowtex-20, $\mathrm{SiO}_{2}$

$=20 \mathrm{wt} \%)$ and an aqueous solution of sodium hydroxide $\left(0.58 \mathrm{~g} / 63 \mathrm{~cm}^{3}\right)$. HDTMABr

(C16), tetradecyltrimethylammonium bromide (C14), dodecyltrimethylammonium

bromide $(\mathrm{C} 12)$, decyltrimethylammonium bromide $(\mathrm{C} 10)$ or octyltrimethylammonium

chloride (C8) was used as the structure-directing agent. The resulting mixture was

stirred for $2 \mathrm{~h}$, followed by a hydrothermal treatment at $413 \mathrm{~K}$ for $48 \mathrm{~h}$ in an autoclave.

The obtained slurry was filtered, washed with water, dried at $353 \mathrm{~K}$ and calcined at 873

$\mathrm{K}$ for $6 \mathrm{~h}$ in a dry air stream. Thus obtained MCM-41 samples are denoted as

Cn-MCM-41(A), where $n$ indicates the length of the alkyl chain of the surfactant used

in the preparation (C16-MCM-41 may simply be denoted as MCM-41), while (A)

indicates that the Si source is the colloidal silica. The molar ratio was 1.0 surfactant :

7.0 $\mathrm{SiO}_{2}: 2.0 \mathrm{NaOH}: 385 \mathrm{H}_{2} \mathrm{O}$. On the other hand, the sample using TEOS (10.0 g) as

the Si source was also prepared in the similar manner described above. Those samples

are designated as MCM-41(B). The molar ratio was 1.0 surfactant : $6.7 \mathrm{SiO}_{2}: 2.0$

$\mathrm{NaOH}: 385 \mathrm{H}_{2} \mathrm{O}$. The MCM-41(B) samples containing Al were prepared by adding 
sodium aluminate (Wako Chemical Co.) to the starting reaction mixture. The Al-containing MCM-41(B) samples are denoted as $\mathrm{Al}(x)-\mathrm{MCM}-41(\mathrm{~B})$, where $x$ indicates the amount of added $\mathrm{Al}(\mathrm{wt} \%)$.

In addition to those mesoporous silicates, conventional silica (JRC-SIO-9A, denoted as $\mathrm{SiO}_{2}$ ) and silica-alumina (JRC-SAH-1, denoted as $\mathrm{SA}$ ), both supplied from the Catalysis Society of Japan, were used as reference host materials. The $\mathrm{SiO}_{2}$ and $\mathrm{SA}$ have the surface area of $336 \mathrm{~m}^{2} / \mathrm{g}$ and $511 \mathrm{~m}^{2} / \mathrm{g}$, respectively, both without mesoporous structure. The $\mathrm{Al}$ contents of them are $2.3 \mathrm{ppm}$ and $15.1 \mathrm{wt} \%$, respectively.

The structure of the mesoporous silicates was estimated by XRD and nitrogen adsorption isotherms. The XRD patterns were measured with PANalytical X'Pert PRO apparatus with $\mathrm{CuK} \alpha$ as an X-ray source. Shimadzu TriStar3000 apparatus was used to carry out the nitrogen adsorption experiments. The specific surface area and the pore diameter of each sample were estimated by BET and BJH methods, respectively.

The acid strength of the inorganic host materials was estimated using Hammett indicator according to the literature [37-39]. After heating at $773 \mathrm{~K}$ for $2 \mathrm{~h}$ to remove adsorbed water, the host material samples were mixed with the dried isooctane solution 
of each indicator. The change in the color of the samples was observed to determine the acid strength.

The amount of Al included in the host materials was estimated by ICP analysis after the HF treatment of the samples. The amount of Si was estimated by X-ray fluorescence analysis applying the glass beads method.

The flavylium dyes used in this study are shown in Scheme 1. FV1 has a methoxy group at 4'-position. In addition to the methoxy group, FV2 and FV3 have a hydroxyl group at 7-position. FV3 further has a phenyl group at 4-position. Despite the difference in the substituents, all these flavylium dyes exhibit yellow color in an acidic solution. However, FV2 and FV3 show red shift of the absorption band in a weakly basic solution, which is due to the dissociation of a proton from the hydroxyl group [40].

The complexation of the FV dyes with the inorganic hosts was carried out as follows. The FV dye (2 mg) was dissolved in aqueous ethanol (water : ethanol $=7: 3$ ), and 200 mg of the inorganic host material was mixed with the solution. The mixture was kept in the dark for 3 days to reach the adsorption equilibrium, followed by filtration to separate the composite sample. Hereafter, the composite samples are referred to as 
FVx/host; for example, the sample composed of FV1 and FSM-16 will be denoted as FV1/FSM-16. The adsorption amount of FV was estimated from the difference in the absorbance of the filtrate and the initial FV solution. Thus calculated adsorption amount of FV was about 7 to $8 \mathrm{mg} / \mathrm{g}$ in FV/FSM-16 or FV/MCM-41(A), and about $5 \mathrm{mg} / \mathrm{g}$ in FV/MCM-41(B), FV/HMS or FV/SiO 2 , irrespective of the type of the FV series.

The light fastness of the samples was determined as follows. A $100 \mathrm{~W}$ halogen lamp (SCHOTT, Megalight 100) equipped with a two-way branched light guide was used as a visible light source. The powdered sample was set in a cell with a glass window and irradiated at the position of $1.5 \mathrm{~cm}$ from the light. The degradation of the sample was estimated from the change in the absorption of diffuse reflectance UV-vis spectra. The UV-Vis spectra were recorded using JASCO V-550 spectrophotometer equipped with ISV-469 integration-sphere attachment. When the light fastness of the sample should be measured under the $\mathrm{O}_{2}$ rich condition, two cells containing an identical sample were held in the gas barrier film, one of which was filled with pure $\mathrm{O}_{2}$ and the other was packed in the air, followed by irradiation with the visible light. 


\section{Results and Discussion}

UV-Vis spectra of the composites

We first collected UV-vis spectra of the composites of each FV dye and inorganic host material. The diffuse reflectance UV-vis spectra of the composite samples are shown in Figure 1. In every spectrum a clear peak was observed at $440 \mathrm{~nm}$ to $470 \mathrm{~nm}$, which was assigned to the absorption of the FV derivatives [25]. In some cases, in addition to the main peak, a sub peak was seen in the shorter wavelength region of about $350 \mathrm{~nm}$, indicating the existence of the molecules adsorbed on the host after translation to the corresponding 2-hydroxychalcones [29].

While the peak position of the FV1 samples was almost the same, the composites of FV2 showed a peak shift caused by the difference in the host materials. Namely, the peak wavelength of FV2/FSM-16 or FV2/MCM-41(A) samples was in shorter region than that of FV2/MCM-41(B), FV2/HMS or FV2/SiO 2 . Such a peak shift was also seen in the composite of FV3; the peak position was red-shifted in FV3/MCM-41(B), 
FV3/HMS or FV3/SiO 2 compared to FV3/FSM-16 or FV3/MCM-41(A). The peak shift observed in the several composites of FV2 or FV3 seemed due to the dissociation of the phenolic hydrogen of FV2 or FV3. Figure 2 shows the UV-vis absorption spectra of FV1, FV2 and FV3 in aqueous ethanolic solution at different $\mathrm{pH}$ conditions. In the spectra of FV2 and FV3, the peak wavelength was shifted to longer region as the $\mathrm{pH}$ value was larger, while the peak was not shifted in the spectra of FV1. These spectral changes were fully reversible, indicating the reversible conversion of the FV to other forms. Considering these results, it is suggested that the solid acidity of MCM-41(B), HMS and $\mathrm{SiO}_{2}$ is weaker than that of FSM-16 and MCM-41(A).

Degradation of the dye under photoirradiation

The light fastness of the FV composite samples was investigated by irradiation of visible light to each sample. As a typical example, Figure 3 represents the UV-vis absorption spectral change in FV1/FSM-16 and $\mathrm{FV} 1 / \mathrm{SiO}_{2}$ during photoirradiation for 8 h. Although the absorption of $\mathrm{FV} 1 / \mathrm{SiO}_{2}$ was more exceedingly lost than FV1/FSM-16, 
the photoirradiation diminished the absorption of both samples. However, both the peak position and the spectral shape were kept during irradiation. The spectral change during irradiation in other samples, including FV2 and FV3 samples, showed almost the same tendency, and no other new band appeared during irradiation of all samples. These results indicate that the decrease in the absorption means a simple degradation of the dye molecules by irradiation, rather than a photoisomerization or another photoreaction of the dye.

Figure 4 represents the change in the absorption of the composite samples. The composites of every FV and FSM-16 or MCM-41(A) exhibited excellent photostability. On the other hand, similar mesoporous silicas such as MCM-41(B) or HMS showed poor effect to stabilize the adsorbed FV against irradiation, and the stability of the composite is almost the same level as that of $\mathrm{FV} / \mathrm{SiO}_{2}$. This result suggests that some properties of the mesoporous silicas play an important role in the enhancement of the stability of the adsorbed FV. However, the regularity of the mesopore has nothing to do with the enhancement of the stability, because MCM-41(B) with highly ordered hexagonal mesopores exhibited similar stabilization effect as HMS, which had 
randomly ordered wormhole-type pores. It should be noted that MCM-41(B), HMS and $\mathrm{SiO}_{2}$, which were not effective to stabilize the adsorbed FV, were suggested to be less acidic than FSM-16 and MCM-41(A), as described above.

Acidic property of mesoporous silicas

It is generally known that flavylium ions (both natural and synthetic) are stable in an acidic solution [29, 30, 41, 42]. On the other hand, when adsorbed on MCM-41(B), HMS and $\mathrm{SiO}_{2}, \mathrm{FV} 2$ and FV3 showed the peak shift based on the dissociation of a phenolic hydroxyl group, as shown in Figure 1 and 2. This fact suggests the weak acidity of those host materials. In addition, the stability of FV/MCM-41(B), FV/HMS or $\mathrm{FV} / \mathrm{SiO}_{2}$ against irradiation was lower than that of FV/FSM-16 or FV/MCM-41(A). These results suggest the relationship between the solid acidity of the inorganic hosts and photostability of the composite samples.

Therefore, we investigated the acid strength of those host materials using the Hammett indicator. The results are represented in Table 1. The $\mathrm{pKa}$ values of FSM-16 and 
MCM-41(A) were $c a .-3.0$ and -5.6 respectively, indicating that they were moderately acidic materials [39]. On the other hand, the pKa values of MCM-41(B), HMS and $\mathrm{SiO}_{2}$ were all larger than 1.5 , indicating that they were not acidic. Taking into consideration that acidic FV/FSM-16 and FV/MCM-41(A) exhibited superior stability than non-acidic FV/MCM-41(B), FV/HMS or $\mathrm{FV} / \mathrm{SiO}_{2}$, we can guess that the acidity of the mesoporous host materials greatly influences the enhancement of the photostability of the composite samples.

Since the impurity of aluminum is known to enhance the solid acidity of mesoporous silicates [43], we tried to estimate the amount of $\mathrm{Al}$ content in our mesoporous silica samples. Table 2 represents the determined $\mathrm{Al}$ content in our mesoporous silicas and their source materials. It can be seen in Table 2 that acidic FSM-16 and MCM-41(A) contained considerable amount of Al, whereas non-acidic MCM-41(B) did not. As the source materials of FSM-16 and MCM-41(A) were also found to contain significant amount of $\mathrm{Al}$, we judged that the $\mathrm{Al}$ included in the mesoporous silicas derived from their source materials. From these, it can be concluded that the Al impurity in the mesoporous silicas will work as an acid site and enhance the light fastness of the 
adsorbed FV.

Next, we prepared the MCM-41 samples using TEOS as a Si source and adding Al intentionally. The Al content of these Al-MCM-41(B) samples is in consistence with the introduced $\mathrm{Al}$ amount, as shown in Table 2 . We confirmed by acid-base titration with $\mathrm{NH}_{3}$ that the amount of acid site increased along with the $\mathrm{Al}$ content, showing that the acid site was due to the Al. However, the acidity of Al-MCM-41(B) was almost the same as that of MCM-41(A) irrespective of the $\mathrm{Al}$ content ( $\mathrm{pKa} \sim-5.6)$, as represented in Table 1.

Table 3 indicates the light fastness data of FV/MCM-41 samples containing various amount of $\mathrm{Al}$, given as a retention ratio of the light absorption after visible light irradiation for $8 \mathrm{~h}$. While FV/MCM-41(B) samples containing very small amount of Al exhibited poor stability, each of FV/Al-MCM-41(B) samples showed improved light fastness, which was almost the same as that of FV/MCM-41(A). This result further supports that the enhancement of the stability of FV was brought about by the $\mathrm{Al}$ impurity.

Although the stability of FV1/Al-MCM-41(B) was sufficient in all range of Al content 
studied here, the stability of FV2/Al(0.07)-MCM-41(B) and FV3/Al(0.07)-MCM-41(B) was insufficient compared to FV/Al(0.13)-MCM-41(B) and FV/Al(0.20)-MCM-41(B). This result seems strange at a glance, because FV1 more easily loses its color than FV2 and FV3 under weakly acidic condition, as shown in Figure 2. However, as discussed above, the acidity of Al-MCM-41(B) was found to be independent of the Al content. Therefore, this result is not inconsistent with the stability order of the FV series. On the other hand, this result can be rephrased that FV1 was stabilized even by the small amount of Al impurity, whereas FV2 and FV3 required more amount of Al to be stabilized. When we carried out the adsorption of FV2 and FV3 onto the inorganic hosts, the color of the FV solutions indicated that FV2 and FV3 were in a quinonoid form releasing the proton of their hydroxyl group. After adsorption, FV2/MCM-41 or FV3/MCM-41 showed the color of the flavylium form with the proton of the hydroxyl group. Therefore, we speculated that part of the acid site in the mesoporous silicates might be consumed for protonation of the FV2 and FV3 in the quinonoid form, resulting in the requirement of extra $\mathrm{Al}$ content. 
Effect of mesopore

In order to confirm the effect of the mesopore on the stability of adsorbed FV, we prepared the composite of FV and silica-alumina (SA), and checked its photostability. As shown in Table 1, the acidity of SA was extremely stronger than MCM-41(A). Table 4 represents the light fastness data of FV/MCM-41(A) and FV/SA. The stability of both FV1/SA and FV2/SA was inferior to that of FV/MCM-41(A). Since the strongly acidic SA did not exhibit enough effect on the stabilization of FV, we can conclude that not only the solid acidity but also the mesopore is essential to improve the stability of FV. In other words, the FV dye molecules are not stabilized until they are incorporated into the mesopore.

We then measured the photostability of FV2 adsorbed on MCM-41(A), MCM-41(B) and $\mathrm{SiO}_{2}$ under $\mathrm{O}_{2}$ rich condition, and calculated the fading rate constant, based on the assumption that the decoloration was a first order reaction. Table 5 shows the fading rate constant of FV2/MCM-41(A), FV2/MCM-41(B) and $\mathrm{FV} 2 / \mathrm{SiO}_{2}$ under irradiation in $\mathrm{O}_{2}$ rich and ambient conditions. From this result, we can see that the fading rate of 
$\mathrm{FV} 2 / \mathrm{SiO}_{2}$ was remarkably promoted under $\mathrm{O}_{2}$ rich condition, whereas those of FV2/MCM-41(A) and FV2/MCM-41(B) were not so drastically promoted even in $\mathrm{O}_{2}$ rich atmosphere. Since the fading rate of MCM-41(B) was in the same level as that of $\mathrm{SiO}_{2}$, the difference in the promotion of the fading rate under $\mathrm{O}_{2}$ atmosphere should not derive from the difference in the fading rate itself, but from the difference whether the host materials were mesoporous or not. Considering that the degradation and fading of the dye molecules under irradiation are often thought due to the reaction with oxygen in the air [44], we can say that FV dye molecules incorporated in the mesopore are stabilized against irradiation because it is hard for them to be influenced by oxygen. Thus, because of the steric hindrance, incorporation of the dye molecules into the mesopore will diminish the attack of the oxygen which will come from the outside of the mesopore by diffusion, and consequently will enhance the stability of FV.

As for the non-acidic host materials, the light fastness of nonporous $\mathrm{FV} 2 / \mathrm{SiO}_{2}$ was higher than mesoporous FV2/MCM-41(B) in the air. As seen in Figure 4, similar trend can be found between the other FV/MCM-41(B), FV/HMS and $\mathrm{FV} / \mathrm{SiO}_{2}$. This fact suggests the difference in the adsorption state of FV in FV/MCM-41(B), FV/HMS and 
$\mathrm{FV} / \mathrm{SiO}_{2}$, although all those hosts are made from silica component. This seems to derive from the difference in the surface area. Since $\mathrm{SiO}_{2}$ had smaller surface area $\left(336 \mathrm{~m}^{2} / \mathrm{g}\right)$ than MCM-41(B) or HMS $\left(\sim 1000 \mathrm{~m}^{2} / \mathrm{g}\right)$, the surface density of FV on the hosts differed from each other, as shown in Table 5. Considering this, we can make a speculation that multilayer adsorption of FV may partly occur on the local surface of $\mathrm{SiO}_{2}$, resulting in the apparent higher stability of $\mathrm{FV} / \mathrm{SiO}_{2}$ against irradiation [16], although we could not observe any shift in the UV-vis spectra caused by the dye aggregate.

Effect of pore diameter

We prepared the MCM-41 whose pore diameter was controlled by changing the alkyl chain length of the surfactant from 16 to 8 . Several properties of the $\mathrm{C} n-\mathrm{MCM}-41(\mathrm{~A})$ series are shown in Table 6. Since all the samples had a large specific surface area and sufficient pore volume, it can be said that all $\mathrm{Cn}$-MCM-41(A) samples had a mesoporous structure. As was expected, the pore diameter became distinguishably 
smaller as the alkyl chain length is shorter. On the other hand, there was little difference in the acid strength of $\mathrm{Cn}$-MCM-41(A) samples estimated by Hammett indicator. Namely, the pKa value of C8-MCM-41(A) was around -3.0, and the values of other Cn-MCM-41(A) samples were about -5.6.

The light fastness of FV2/Cn-MCM-41(A) samples is also shown in the extreme right column of Table 6 . From this result, we can see that the effect of complexation on the stability enhancement became weaker with the decrease in the pore diameter of MCM-41(A). Following is the discussion about the reason why the light fastness was inferior as the pore diameter became smaller.

First, the difference in the adsorption state can be regarded. The decrease in a number of the adsorption site may cause the formation of an aggregate and influence the stability of the adsorbed dye. However, no significant difference was seen in the amount of FV2 adsorbed on each $\mathrm{Cn}$-MCM-41(A). In addition, the UV-vis spectra of each FV2/Cn-MCM-41(A) samples exhibited no significant difference in the spectral shape suggesting the existence of the aggregate, as shown in Figure 5. These results suggested that the number of the adsorption site was similar to each other, and that the formation 
of an aggregate was not plausible on any samples. Therefore, it was concluded that the adsorption state of FV2 was identical through the $\mathrm{C} n-\mathrm{MCM}-41(\mathrm{~A})$ series.

Second, it is able to think that the adsorption of FV2 on the external surface of $\mathrm{Cn}$-MCM-41(A) became major as the pore diameter was smaller, since the pore volume decreased along with the decrease in the pore diameter. The stabilization effect of the mesoporous silicas is expected to be weak on the FV adsorbed on the external surface. However, when the FV2/Cn-MCM-41(A) samples were washed with ethanol, more amount of FV2 eluted from the samples with larger pore diameter rather than with smaller one. Therefore, we should exclude the possibility that external surface adsorption of FV2 was prominent on the $\mathrm{C} n$-MCM-41(A) with small pore volume.

After all, the reason why the photostability of FV2/Cn-MCM-41(A) dropped down by the decrease in the pore diameter is still unclear and now under investigation.

\section{Conclusion}

The stability of flavylium dye against visible light irradiation was enhanced by the 
incorporation into the pore of mesoporous silicas exhibiting solid acidity caused by a small amount of Al. The flavylium dyes having a hydroxyl group required more amount of acid site to be stabilized in the mesoporous silicas. As the contact between the dye and oxygen molecule was diminished in the mesopore, the photostability of the flavylium dye was enhanced by incorporation into the mesoporous silicas. The enhancement of the photostability was small when the dye was incorporated into the mesoporous silicas with small pore diameter, although the reason has not been clarified yet.

References

[1] M. Jose-Yacaman, L. Rendon, J. Arenas, M.C.S. Puche, Science 273 (1996) 223.

[2] P. Gomez-Romero, C. Sanchez, New J. Chem. 29 (2005) 57.

[3] J. Bujdak, N. Iyi, T. Fujita, Colloid. Surf. A 207 (2002) 207.

[4] T. Endo, N. Nakada, T. Sato, M. Shimada, J. Phys. Chem. Solids 50 (1989) 133.

[5] T. Endo, M. Shimada, Stud. Surf. Sci. Catal. 60 (1991) 189. 
[6] T. Saito, K. Fukui, Y. Kodera, A. Matsuyama, H. Nishimura, Y. Inada, Dyes Pigments 65 (2005) 21.

[7] C.B. Sunwar, H. Bose, J. Colloid Interface Sci. 136 (1990) 54.

[8] H. Laguna, S. Loera, I.A. Ibarra, E. Lima, M.A. Vera, V. Lara, Microporous Mesoporous Mater. 98 (2007) 234.

[9] E. Lima, M.J. Martinez-Ortiz, E. Fregoso, J. Mendez-Vivar, Stud. Surf. Sci. Catal. 170B (2007) 2110.

[10] G. Calzaferri, Chimia 52 (1998) 525.

[11] W.F. Hölderich, N. Röhrlich, P. Bartl, L. Chassot, Phys. Chem. Chem. Phys. 2 (2000) 3919.

[12] R. Hoppe, G. Schulz-Ekloff, D. Woehrle, C. Kirschhock, H. Fuess, Stud. Surf. Sci. Catal. 84 (1994) 821.

[13] T. Itoh, K. Yano, Y. Fukushima, J. Am. Chem. Soc. 124 (2002) 13437.

[14] M. Shibata, H. Hotta, T. Suzuki, S. Valange, Z. Gabelica, Chem. Lett. (1999) 1291. 
[15] Z. Gabelica, M. Shibata, H. Hotta, T. Suzuki, S. Valange, Stud. Surf. Sci. Catal. $140(2001) 279$.

[16] Z. Gabelica, S. Valange, M. Shibata, H. Hotta, T. Suzuki, Microporous Mesoporous Mater. 44-45 (2001) 645.

[17] K. Moller, T. Bein, Chem. Mater. 10 (1998) 2950.

[18] K. Moller, T. Bein, Stud. Surf. Sci. Catal. 117 (1998) 53.

[19] H. Yamashita, A. Tanaka, M. Nishimura, K. Koyano, T. Tatsumi, M. Anpo, Stud. Surf. Sci. Catal. 117 (1998) 551.

[20] G. Telbiz, O. Shvets, S. Boron, V. Vozny, M. Brodyn, G. Stucky, Stud. Surf. Sci. Catal. 135 (2001) 3564.

[21] M. Ogawa, J. Photochem. Photobiol. C 3 (2002) 129.

[22] I. Oda, K. Hirata, S. Watanabe, Y. Shibata, T. Kajino, Y. Fukushima, S. Iwai, S. Itoh, J. Phys. Chem. B 110 (2006) 1114.

[23] G.A. Iacobucci, J.G. Sweeny, Tetrahedron 39 (1983) 3005.

[24] D. Amic, D. Davidovic-Amic, D. Beslo, B. Lucic, N. Trinajstic, J. Chem. Inf. Comput. Sci. 39 (1999) 967. 
[25] R. Matsushima, K. Miyakawa, M. Nishihata, Chem. Lett. (1988) 1915.

[26] R. Matsushima, S. Fujimoto, K. Tokumura, Bull. Chem. Soc. Jpn. 74 (2001) 827.

[27] R. Matsushima, H. Mizuno, H. Itoh, J. Photochem. Photobiol. A 89 (1995) 251.

[28] R. Matsushima, H. Mizuno, A. Kajiura, Bull. Chem. Soc. Jpn. 67 (1994) 1762.

[29] R.A. McClelland, S. Gedge, J. Am. Chem. Soc. 102 (1980) 5838.

[30] R.A. McClelland, G.H. McGall, J. Org. Chem. 47 (1982) 3730.

[31] D.B. Devine, R.A. McClelland, J. Org. Chem. 50 (1985) 5656.

[32] K. Torskangerpoll, O.M. Andersen, Food Chem. 89 (2004) 427.

[33] Y. Kohno, R. Hoshino, R. Matsushima, Y. Tomita, K. Kobayashi, J. Jpn. Soc. Colour Mater. 80 (2007) 6.

[34] S. Inagaki, A. Koiwai, N. Suzuki, Y. Fukushima, K. Kuroda, Bull. Chem. Soc. Jpn. 69 (1996) 1449.

[35] N. Marín-Astorga, G. Pecchi, T.J. Pinnavaia, G. Alvez-Manoli, P. Reyes, J. Mol. Cat. A 247 (2006) 145.

[36] M. Iwamoto, T. Abe, Y. Tachibana, J. Mol. Cat. A 155 (2000) 143. 
[37] H.A. Benesi, J. Am. Chem. Soc. 78 (1956) 5490.

[38] H.A. Benesi, J. Phys. Chem. 61 (1957) 970.

[39] T. Yamamoto, T. Tanaka, T. Funabiki, S. Yoshida, J. Phys. Chem. B 102 (1998) 5830.

[40] J.C. Lima, I. Abreu, R. Brouillard, A.L. Macanita, Chem. Phys. Lett. 298 (1998) 189.

[41] R. Brouillard, B. Delaporte, J. Am. Chem. Soc. 99 (1977) 8461.

[42] R. Brouillard, J.-E. Dubois, J. Am. Chem. Soc. 99 (1977) 1359.

[43] S. Inagaki, Y. Yamada, Y. Fukushima, Stud. Surf. Sci. Catal. 105 (1997) 109.

[44] Y. Usui, K. Ito, M. Koizumi, Bull. Chem. Soc. Jpn. 38 (1965) 1015. 
Table 1 Acid strength of mesoporous silicas, silica and silica-alumina samples

\begin{tabular}{cc}
\hline Sample & $\mathrm{pKa}^{\text {a) }}$ \\
\hline FSM-16 & $\sim-3.0$ \\
MCM-41(A) & $\sim-5.6$ \\
MCM-41(B) & $>+1.5$ \\
$\mathrm{HMS}$ & $>+1.5$ \\
$\mathrm{SiO}_{2}$ & $>+1.5$ \\
$\mathrm{SA}$ & $<-5.6$ \\
$\mathrm{Al}(0.07)-\mathrm{MCM}-41(\mathrm{~B})$ & $\sim-5.6$ \\
$\mathrm{Al}(0.13)-\mathrm{MCM}-41(\mathrm{~B})$ & $\sim-5.6$ \\
$\mathrm{Al}(0.20)-\mathrm{MCM}-41(\mathrm{~B})$ & $\sim-5.6$ \\
\hline
\end{tabular}

a) determined by Hammett indicator 
Table 2 Determination of Al content in mesoporous silicas and their source materials

\begin{tabular}{ccc}
\hline Sample & Si source & Al content $(\mathrm{wt} \%)$ \\
\hline FSM-16 & water glass & 0.27 \\
MCM-41(A) & colloidal silica & 0.15 \\
MCM-41(B) & TEOS & 0.005 \\
HMS & TEOS & $n . d .^{\text {a) }}$ \\
Al(0.07)-MCM-41(B) & TEOS & 0.07 \\
Al(0.13)-MCM-41(B) & TEOS & 0.15 \\
Al(0.20)-MCM-41(B) & TEOS & 0.27 \\
\hline water glass & - & $0.05^{\text {b) }}$ \\
colloidal silica & - & 0.20 \\
\hline
\end{tabular}

a) not determined , b) The presence of other components brought about such a low value. $\mathrm{Si} / \mathrm{Al}=338$.

This value can be converted to " $0.13 \mathrm{wt} \%$ of $\mathrm{Al}$ ". 
Table 3 Light fastness of FV/MCM-41 samples containing various amounts of Al

\begin{tabular}{clll}
\hline \multirow{2}{*}{ Sample } & \multicolumn{3}{c}{ Retention ratio, $A / A_{0}$ a) } \\
\cline { 2 - 4 } MCM-41(A) & FV1 & FV2 & FV3 \\
\hline MCM-41(B) & 0.88 & 0.85 & 0.79 \\
Al(0.07)-MCM-41(B) & 0.85 & 0.13 & 0.53 \\
Al(0.13)-MCM-41(B) & 0.82 & 0.65 & 0.57 \\
Al(0.20)-MCM-41(B) & 0.75 & $\sim 1.0$ & $\sim 1.0$ \\
\hline
\end{tabular}

a) retention ratio of the light absorption after visible light irradiation for $8 \mathrm{~h}$ 
Table 4 Light fastness of FV/MCM-41(A) and FV/SA

\begin{tabular}{cll}
\hline & \multicolumn{2}{c}{ Retention ratio, $A / A_{0}{ }^{\text {a) }}$} \\
\cline { 2 - 3 } Sample & FV1 & FV2 \\
\hline MCM-41(A) & 0.88 & 0.85 \\
SA & 0.42 & 0.67 \\
\hline
\end{tabular}

a) retention ratio of the light absorption after visible light irradiation for $8 \mathrm{~h}$ 
Table 5 Comparison of the fading rate constants of several FV2/ host samples under visible light irradiation in ambient or $\mathrm{O}_{2}$ rich atmosphere, together with the surface density of FV on each host

\begin{tabular}{|c|c|c|c|c|}
\hline \multirow{2}{*}{ Sample } & \multicolumn{2}{|c|}{ Fading rate constant $\left(h^{-1}\right)^{a)}$} & \multirow{2}{*}{$\begin{array}{c}\text { Rate ratio } \\
\mathrm{O}_{2} / \mathrm{Air}\end{array}$} & \multirow{2}{*}{$\begin{array}{l}\text { Surface density } \\
\left(\mathrm{nm}^{2} / \text { molecule }\right)^{\mathrm{b})}\end{array}$} \\
\hline & Air & $\mathrm{O}_{2}$ rich & & \\
\hline FV2/MCM-41(A) & 0.018 & 0.026 & 1.44 & 64 \\
\hline FV2/MCM-41(B) & 0.120 & 0.191 & 1.60 & 68 \\
\hline $\mathrm{FV} 2 / \mathrm{SiO}_{2}$ & 0.084 & 0.234 & 2.78 & 34 \\
\hline
\end{tabular}

a) based on the assumption that the fading is a first order reaction, b) BET surface area divided by the number of adsorbed FV molecule. 
Table 6. Properties of $\mathrm{C} n$-MCM-41(A) and the light fastness of FV2/Cn-MCM-41(A)

\begin{tabular}{ccccc}
\hline Sample & $\begin{array}{c}\text { Specific } \\
\text { surface area } \\
\left(\mathrm{m}^{2} / \mathrm{g}\right)^{\mathrm{a})}\end{array}$ & $\begin{array}{c}\text { Mean pore } \\
\text { diameter }(\mathrm{nm})^{\mathrm{b})}\end{array}$ & $\begin{array}{c}\text { Pore volume } \\
\left(\mathrm{cm}^{3} / \mathrm{g}\right)^{\mathrm{b})}\end{array}$ & $\begin{array}{c}\text { Retention } \\
\left.\text { ratio, } A / A_{0}{ }^{\mathrm{c}}\right)\end{array}$ \\
\hline C16-MCM-41(A) & 1134 & 2.9 & 0.840 & 0.85 \\
C14-MCM-41(A) & 1104 & 2.6 & 0.721 & 0.71 \\
C12-MCM-41(A) & 1069 & 2.3 & 0.636 & 0.82 \\
C10-MCM-41(A) & 1179 & 1.9 & 0.494 & 0.62 \\
C8-MCM-41(A) & 911 & 1.5 & 0.377 & 0.46 \\
\hline
\end{tabular}

a) BET specific surface area , b) determined by BJH method, c) retention ratio of the light absorption after visible light irradiation for $8 \mathrm{~h}$ 
Figure captions

Figure 1. Diffuse reflectance UV-vis spectra of the composites between [A] FV1, [B] FV2, [C] FV3 and several inorganic hosts. The inorganic hosts were (a) FSM-16, (b) MCM-41(A), (c) MCM-41(B), (d) HMS and (e) $\mathrm{SiO}_{2}$, respectively. The ordinate values were normalized at the maximum value.

Figure 2. UV-Vis absorption spectra of [A] FV1, [B] FV2 and [C] FV3 aqueous ethanolic solution at various $\mathrm{pH}$.

Figure 3. Change in the diffuse-reflectance UV-Vis spectra of [A] FV1/FSM-16 and [B] $\mathrm{FV} 1 / \mathrm{SiO}_{2}$ caused by visible light irradiation. The spectra were recorded at $0,2,4,6,8 \mathrm{~h}$ under continuous irradiation.

Figure 4. Change in the absorption of the composites between [A] FV1, [B] FV2, [C] FV3 and several inorganic hosts. The inorganic hosts were (a) FSM-16, (b) 
MCM-41(A), (c) MCM-41(B), (d) HMS and (e) $\mathrm{SiO}_{2}$, respectively. The ordinate is expressed as the retention ratio of the absorption to the initial value, $A / A_{0}$.

Figure 5. Diffuse reflectance UV-Vis spectra of FV2/Cn-MCM-41(A) samples. The value $n$ differed from 16 to 8 . The ordinate values were normalized at the maximum value. 

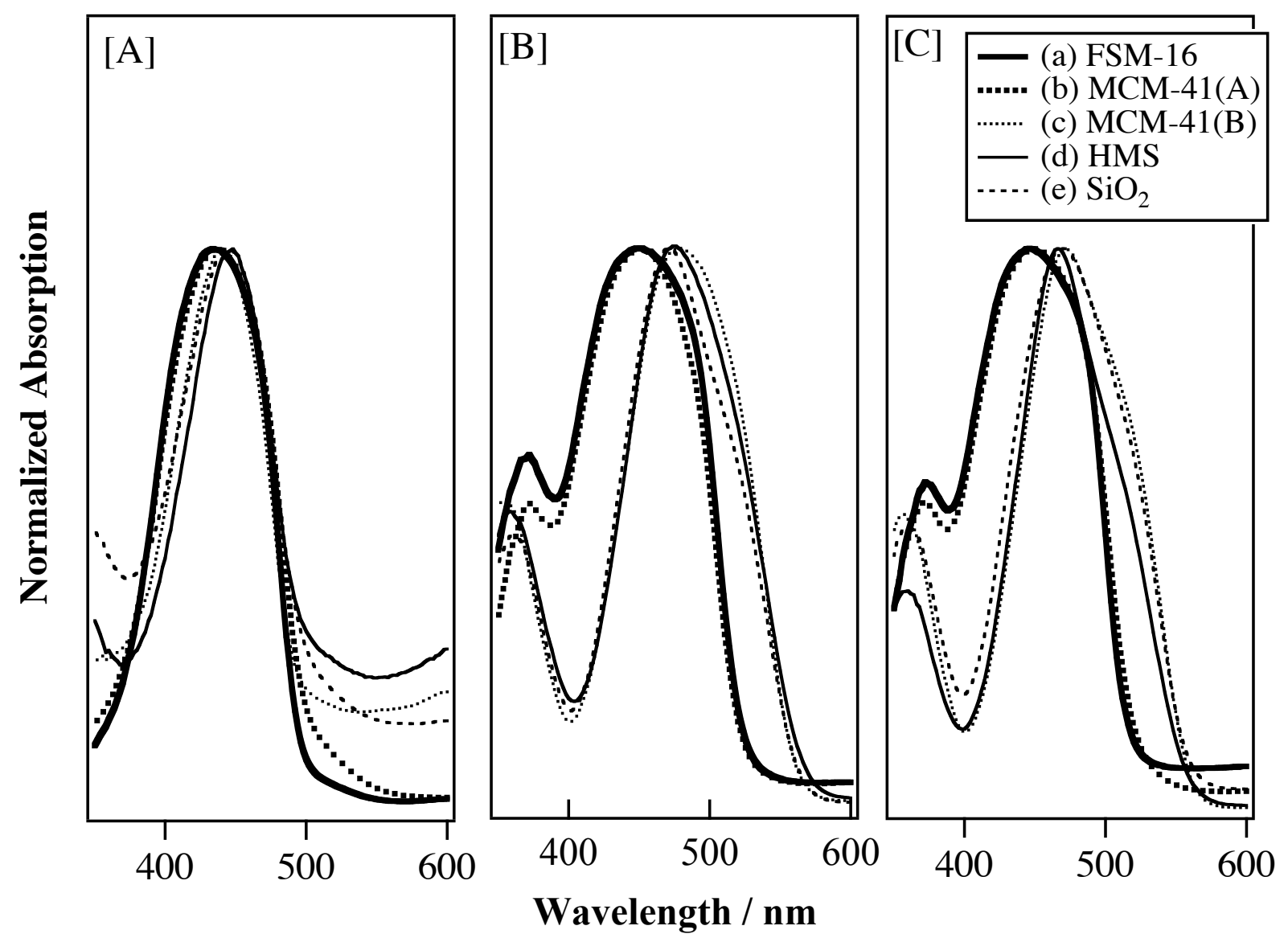

Figure 1. by Yoshiumi Kohno "Enhancement of the photostability of flavylium dye..." 


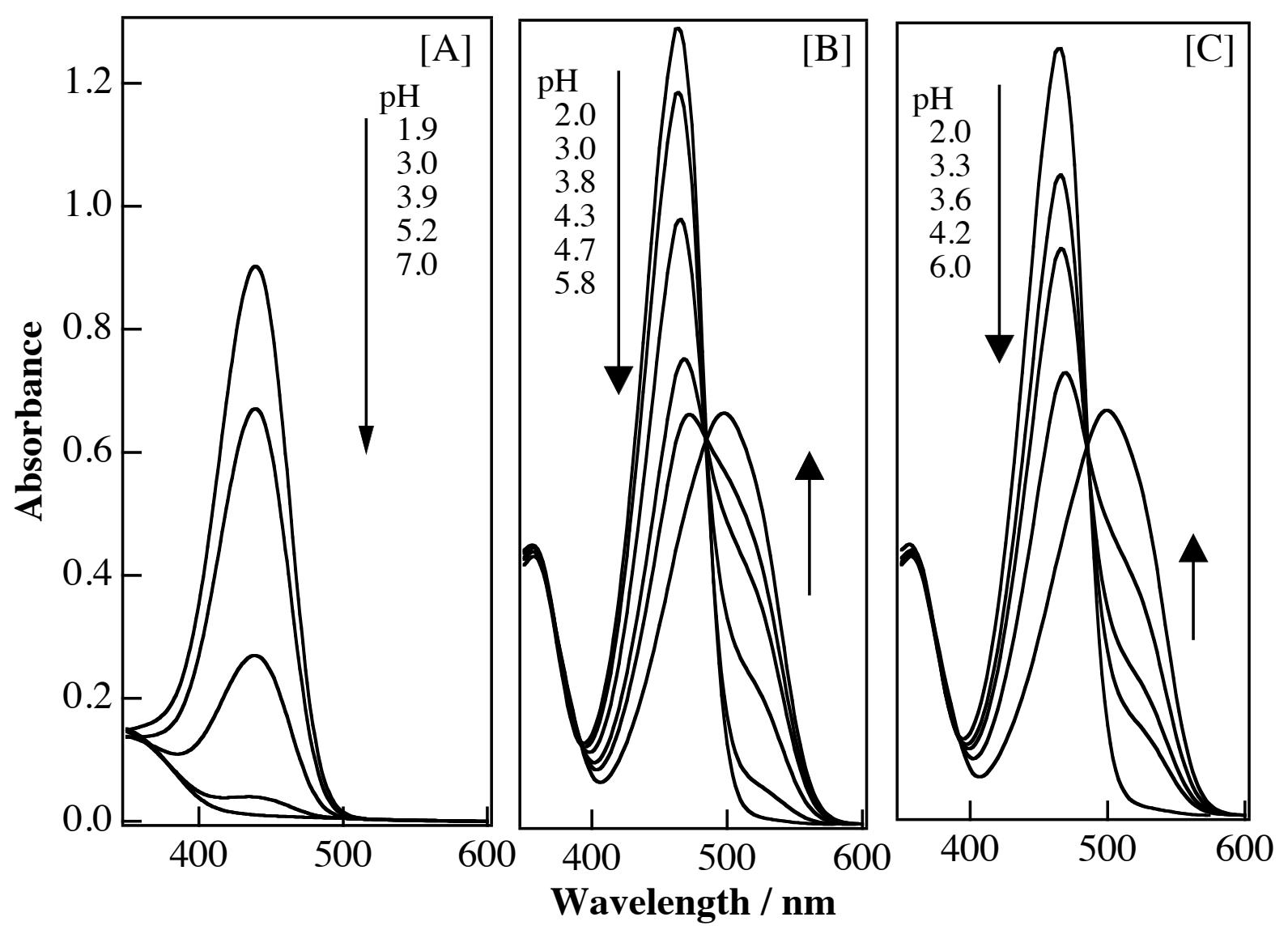

Figure 2. by Yoshiumi Kohno "Enhancement of the photostability of flavylium dye..." 

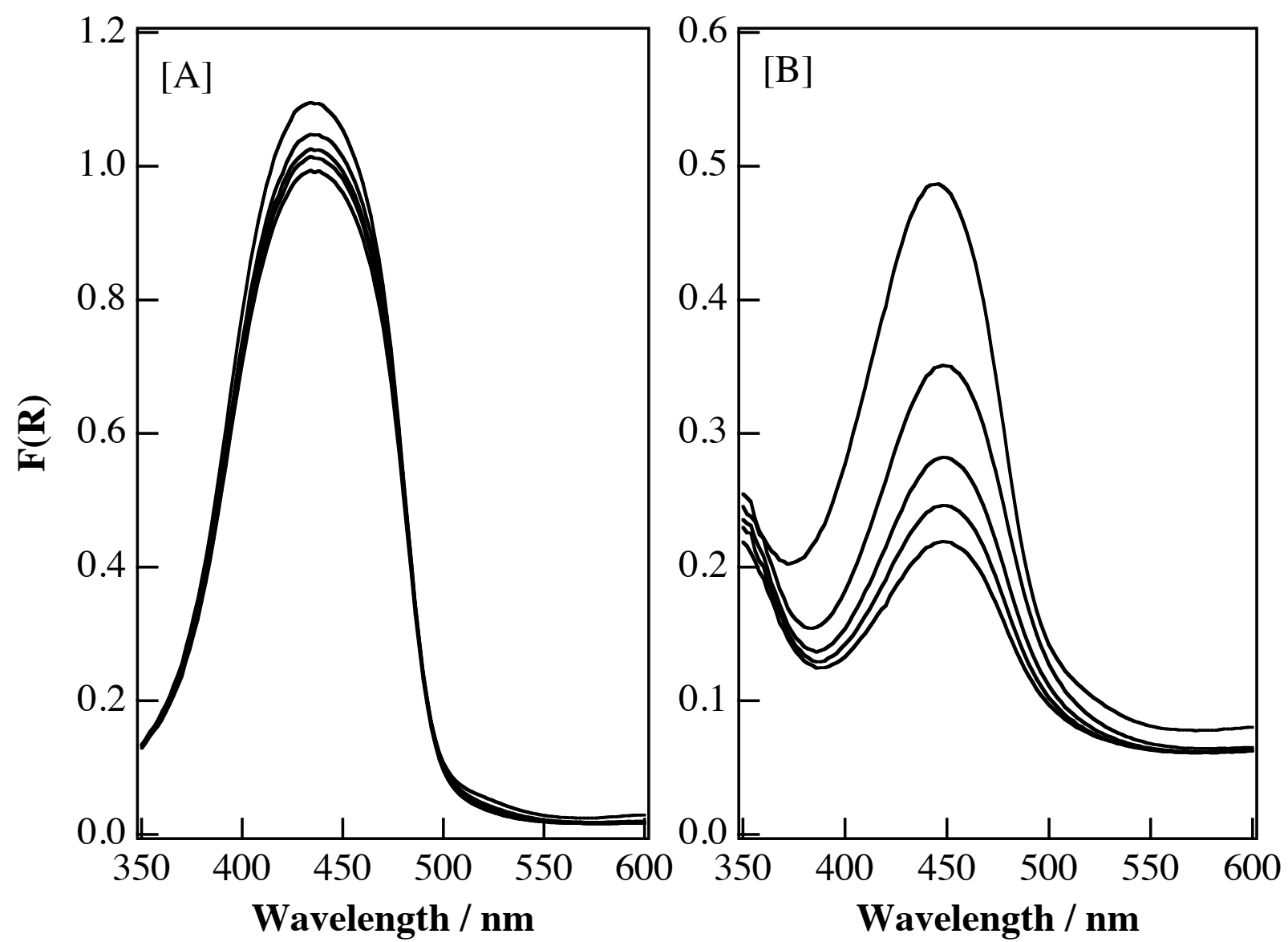

Figure 3. by Yoshiumi Kohno "Enhancement of the photostability of flavylium dye..." 

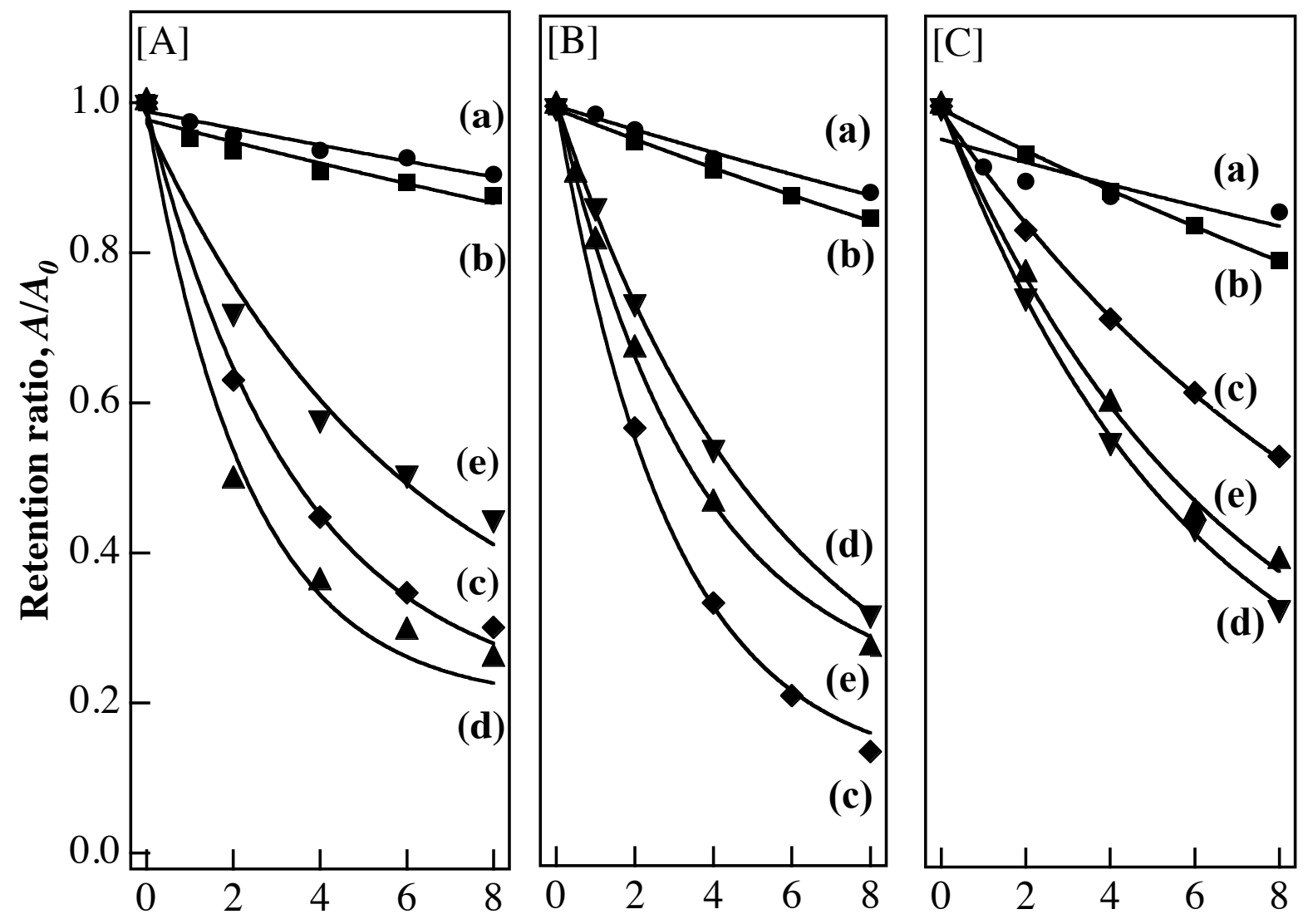

Irradiation time / $\mathbf{h}$

Figure 4. by Yoshiumi Kohno "Enhancement of the photostability of flavylium dye..." 


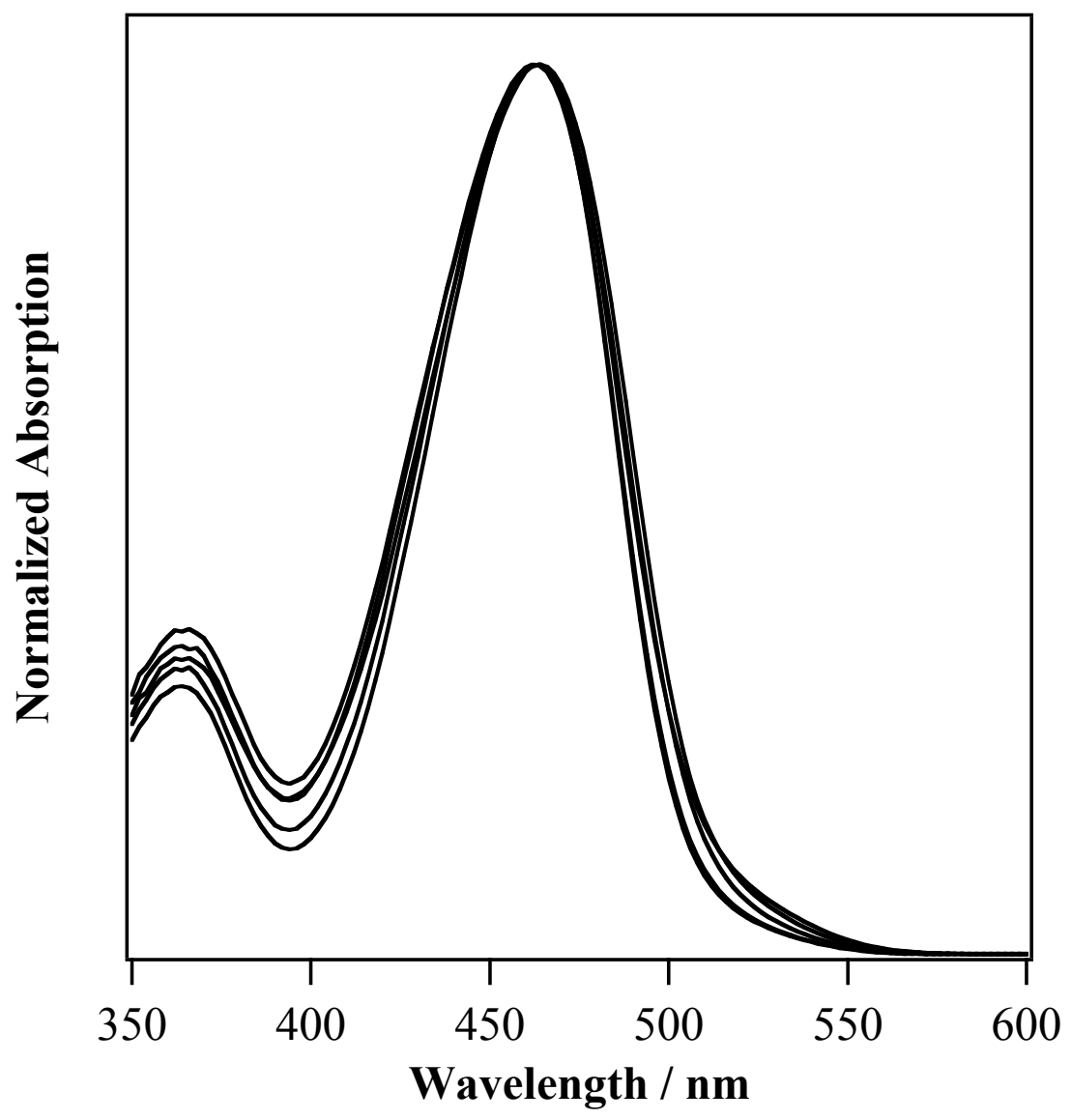

Figure 5. by Yoshiumi Kohno "Enhancement of the photostability of flavylium dye..." 


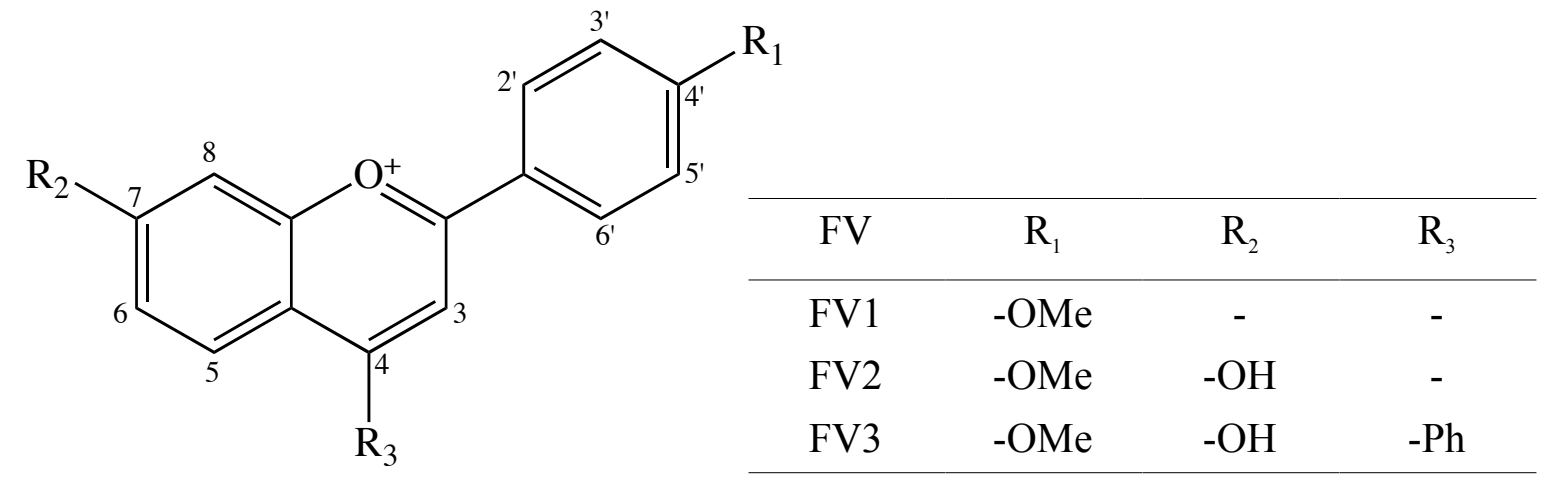

Scheme 1

Scheme 1. by Yoshiumi Kohno "Enhancement of the nhotostahilitv of flavvlium dve..." 